\title{
Metodología de investigación enfocado en ell análisis Cualitativo-Cuantitativo aplicado a los Factores que condicionan la deserción escolar de los alumnos de secundaria Somotillo
}

\author{
Research methodology focused on qualitative analysis - \\ Quantitative applied to the Factors that condition the \\ dropout of students of secondary of the zone $\mathrm{N}^{\circ} 2$, enrolled in \\ year 2015 of the municipality of Somotillo -Chinandega.
}
Ordoñez Pineda, José Joel; Fornos García, Vianey; Editor Academico Prof. Ph.D Angel Sol-Sanchez

José Joel Ordoñez Pineda

vsjoel@post.unanleon.edu.ni

Universidad Nacional Autónoma de Nicaragua.

UNAN-León, Nicaragua

Vianey Fornos García

Universidad Nacional Autónoma de Nicaragua.

UNAN-León, Nicaragua

Editor Academico Prof. Ph.D Angel Sol-Sanchez

sol@colps.mx

Colegio de postgraduados, Mexico

Revista Iberoamericana de Bioeconomía y Cambio Climático

Universidad Nacional Autónoma de Nicaragua, León, Nicaragua ISSN-e: 2410-7980

Periodicidad: Semestral

vol. 3, núm. 5, 2017

czuniga@ct.unanleon.edu.ni

Recepción: 02 Enero 2017

Aprobación: 26 Julio 2017

URL: http://portal.amelica.org/ameli/journal/394/3941752006/

DOI: https://doi.org/10.5377/ribcc.v3i5.5943

Autor de correspondencia: vsjoel@post.unanleon.edu.ni
Resumen: En el presente estudio se investigó de manera concreta y especifica con el propósito de validar y/o experimentar un trabajo para su posterior documentación con artículos bien documentados que son de gran utilidad para la sociedad. Por medio de la investigación cualitativa o cuantitativa que efectúan los investigadores, ya sea éste de acuerdo a su desempeño donde se desenvuelven o resultados obtenidos, muchos de estos son aprovechados por las sociedades, así como otros de ninguna manera. A demás se considera que el análisis en los últimos años, diversos investigadores han definido y estructurado este tipo de metodología como el haz de grandes avances para facilitar el trabajo en documentaciones bibliográfica, estudios de casos, u otros estudios de casos de investigación de naturaleza cuantitativa y cualitativa.

Palabras clave: Metodología, Cuantitativo, Cualitativo, Técnicas.

Abstract: In the present study, it was specifically and specifically investigated with the purpose of validating and / or experiencing a job for subsequent documentation with well-documented articles that are very useful for society. Through the qualitative or quantitative research carried out by the researchers, either according to their performance where they operate or obtained results, many of these are used by societies, as well like others in no way. In addition, it is considered that the analysis in recent years, various researchers have defined and structured this type of methodology as the beam of large advances to facilitate the work in bibliographic documentation, studies of cases, or other research case studies of a quantitative nature and qualitative.

Keywords: methodology, Quantitative, Qualitative, Techniques. 


\section{INTRODUCCIÓN}

En el marco de implementación del presente trabajo de investigación en el afán por efectuar nuevos estudios para poder analizarlos minuciosamente y documentar sus informaciones relacionadas con artículos bibliográficos de otros ensayos.

Los campos de investigación son fundamentales ya que en ellos se emplean distintas técnicas recolección de datos. Estos son primordiales para el estudio que se vaya a realizar, sin importar el caso en estudio, en este artículo se describen la metodología y las técnicas que se emplean en una investigación de carácter cualitativocuantitativo, en la que se describen cada uno de sus análisis, el cual le sirven de guía orientadora al realizar un tipo de investigación.

Para la obtención de datos veraces es primordial que el investigador implemente técnicas de investigación bien definidas y documentadas.

Esta demostrados que, dentro de un estudio de investigación, el investigador debe de hacer el empelo necesario de las técnicas que ha determinado que se necesitan para que el trabajo sea más efectivo y relacionar los otros estudios efectuados en la misma área.

\section{Metodología}

Esta se define como el instrumento del cual depende la técnica que se va implementar para obtener información en una investigación, destacando que siempre debemos de ser cuidadoso al determinar un tipo de estudio aplicado

Según Bogdan y Taylor (1994: 15), opinan que una metodología es "el modo en que enfocamos los problemas y buscamos las respuestas. En las investigaciones en la aplicación de técnicas de investigación y recolección de datos, estos deben aplicados de acuerdo al problema ya que cada uno necesitamos dar una respuesta.

En la utilización de instrumentos en una investigación es de gran importancia, ya que se puede decir afirmar que es el principal factor para obtener los resultados ya sean estos de carácter cualitativo o cuantitativo, siempre fundamentados en el análisis previo a la investigación que se esté o se allá finalizado su estudio.

omando en cuenta que en la metodología se aplican un conjunto de procedimientos que nos sirven de instrumento para lograr los objetivos propuestos de la investigación, por los tanto se puede afirmar que una aplicación de metodología correcta puede basarse en dos procesos metodológicos complementarios: como son el análisis de contenido y estudio de casos.

Bogdan, R. y Tayor, S.J. (1994) plantea que como Introducción a los métodos cualitativos de investigación. Barcelona, Paidós., nos dice que método es cuando nos referimos se siguiendo un componente lógico particular en tanto que se trata de convertir la simple especulación ideológica, filosófica o literaria sobre la sociedad en intentos de comprensión o explicación científica.

Los métodos de investigación deben de ir acompañado de una investigación científica sustentada por el respaldo bibliográfico que debe de llevar toda investigación donde la información debe de ser objetiva y veras.

NotAS DE AUTOR

vsjoel@post.unanleon.edu.ni 


\section{Técnicas de investigación y recolección de datos.}

Esta se procesos específicos a través de los cuales se reúnen y ordenan los datos antes de someterlos a las operaciones lógicas o estadísticas. Gonzales Rios, (1997) Técnica se refieren a los elementos del método científico y no deben confundirse con los métodos; sin embargo, su utilización a veces es confusa y llega a hablarse de métodos de investigación cuando en realidad se refiere a técnicas de recogida o análisis de datos. Ejemplos de técnicas de recolección de datos son "las entrevistas, la encuesta, el grupo de discusión, los experimentos o las escalas de actitudes.

En la investigación utilizare técnica de recolección de datos como: Encuesta, entrevistas directas y grupos focales de orden cualitativo y cuantitativo. La cual se hará con el fin de obtener resultados concretos y eficaces en el trabajo de investigación.

La encuesta. Según Torres, et al., (2006). Existen dos formas de conceptualizar el término encuesta:

a) la encuesta como un instrumento de recogida de datos.

b) La encuesta como un método de investigación.

En el primer caso, la encuesta se considera un procedimiento más de recogida de datos, como los cuestionarios, las entrevistas, las escalas de opinión, los inventarios, etc, y forma parte de la fase de recogida de datos dentro de un método más amplio de investigación, tal como el método experimental, cuasiexperimental o correlacional. En este caso, el término encuesta es sinónimo de cuestionario o entrevista.

En el segundo caso, la encuesta se considera una estrategia de investigación, un procedimiento que implica un proceso completo de investigación, que abarca la formulación del problema, el establecimiento de los objetivos, la selección de los sujetos y el diseño y análisis de datos.

En este segundo caso, el método de encuesta se entiende como una estrategia concreta dentro del método selectivo o correlacional. Según esta segunda conceptualización, se puede definir la metodología de encuestas por dos características fundamentales: Una, como un método no experimental cuya característica principal es la recogida de información en ausencia de manipulación o intervención por parte del experimentador, utilizando procedimientos de observación o medida consistentes y estandarizados para todos los sujetos, de forma que quede garantizada la comparabilidad de los datos.

Onwuegbuzie, et al., (2013): Grupos focales ("FOCUS GROUPS"). Los grupos focales como herramientas de investigación cualitativa

Los grupos focales son ante todo una técnica de investigación cualitativa, donde la discusión grupal se utiliza como un medio para generar entendimiento profundo de las experiencias y creencias de los participantes. Como lo señala (Morgan y Smircich 1980) los grupos focales se planifican en base a tres elementos constitutivos de toda investigación cualitativa:

a) exploración y descubrimiento.

b) b) contexto y profundidad

c) c) interpretación.

Exploración y descubrimiento: los métodos cualitativos son muy útiles cuando se trata de explorar y descubrir. Los grupos focales en este contexto, son usados frecuentemente para aprender sobre opiniones o comportamientos de la gente acerca de lo cual se sabe muy poco o nada.

Partiendo del hecho que el grupo puede llevar a cabo una conversación en torno a lo que interesa a sus miembros, es posible iniciar una exploración acerca de un tema a pesar de que se sabe muy poco respecto de sus características.

Contexto y profundidad: estas dos dimensiones son centrales para comprender el trasfondo que existe detrás de las actitudes de la gente. Los grupos focales llegan a estas dimensiones a través de impulsar en los participantes el deseo de investigar las modalidades y formas mediante las cuales ellos son, al mismo tiempo, semejantes y diferentes unos a otros. 
Los métodos cualitativos en general nos permiten llegar a esta comprensión de por qué las cosas son como son y la dinámica que implica su acontecer

Interpretación: en los grupos focales los participantes tratarán de entenderse mutuamente, y al mismo tiempo los investigadores deberán tratar de comprender por qué dos personas que provienen de contextos tan similares piensan sin embargo tan distinto, así como dos personas que provienen de contextos diferentes piensan sin embargo en forma similar. Estas mismas interrogantes harán que los participantes traten de entenderse mutuamente, lo cual no quiere en todo caso decir que terminarán pensando en forma similar.

\section{El enfoque cuantitativo.}

Sampieri, et al., (2010), plantea que eI enfoque cuantitativo tiene las siguientes características:

1. Que el investigador realiza los siguientes pasos:

a) Plantea un problema de estudio delimitado y concreto. Sus preguntas de investigación versan sobre cuestiones específicas.

b) Una vez planteado el problema de estudio, revisa 10 que se ha investigado anteriormente.

A esta actividad se Ie conoce como la revisión de la literatura.

c) Sobre la base de la revisión de la literatura construye un marco teórico ( Ia teoría que habrá de guiar su estudio). De esta teoría deriva hipótesis (cuestiones que va a probar si son ciertas 0 no).

e) Somete a prueba las hipótesis mediante el empleo de los diseños de investigación apropiados. Si los resultados corroboran las hipótesis 0 son congruentes con estas, se aporta evidencia en su favor. Si se refutan, se descartan Enfoque cuantitativo Usa la recolección de datos para probar hipótesis, con base en la medición numérica y el análisis estadístico, para establecer patrones de comportamiento y probar teorías. En busca de mejores explicaciones y nuevas

hipótesis. AI apoyar las hipótesis se genera confianza en la teoría que las sustenta. Si no es así, se descartan las hipótesis y, eventualmente, la teoría.

i) Para obtener tales resultados el investigador recolecta datos numéricos de los objetos, fenómenos o participantes, que estudia y analiza mediante procedimientos estadísticos.

De este conjunto de pasos, denominado proceso de investigación cuantitativo, se derivan otras características del enfoque cuantitativo que se precisan a continuación:

2. Las hipótesis (por ahora denominémoslas creencias) se generan antes de recolectar y analizar los datos. Por esto se explica que las hipótesis se establecen previamente.

3. La recolección de los datos se fundamenta en la medición (se miden variables 0 conceptos contenidos en las hipótesis). Esta recolección o medición se lleva a cabo al utilizar procedimientos estandarizados y aceptados por una comunidad científica. Para que una investigación sea creíble y aceptada por otros investigadores, debe demostrarse que se siguieron tales procedimientos. Como en este enfoque se pretende medir, los fenómenos estudiados deben poder observarse 0 medirse en el "mundo real).

4. Debido a que los datos son producto de mediciones, se representan mediante números (cantidades) y se deben analizar a través de métodos estadísticos. Dicho de otra manera, las mediciones se transforman en valores numéricos (datos cuantificables) que se analizaron por medio de la estadística.

Los partidarios del análisis cualitativo afirman que la supuesta neutralidad y precisión de medida de los datos cuantitativos no pasan de ser una mera afirmación ideológica, resaltando el escaso valor explicativo de varianza aportado por los cuestionarios con fines estadísticos y ridiculizando el abuso esotérico de fórmulas cada vez más cabalísticas para el logro de unas definiciones de los fenómenos sociales progresivamente alejadas de la realidad social (Ruiz, 1996).

En esencia, no se trata de un simple debate metodológico; la raíz del problema reside en la ontología y epistemología de las investigaciones. Es la controversia clásica que enfrenta las aproximaciones positivistas con las interpretativas, la objetividad frente a la subjetividad (Morgan y Smircich, 1980). 
(Wildemuth, 1993)

Algunos autores han definido los datos cualitativos como aquellos que no son cuantitativos, aquellos que no pueden ser expresados numéricamente (Tesch, 1990).

Considerando que el análisis cuantitativo y cualitativo es fundamental cuando la investigación es documentación bibliográfica o por terminología, se destaca que una de este tipo de análisis determina la información de la otra, porque uno lo interpreta en una determinación numérica y la otra lo asigna con el conocimiento que describió, y pasan de ser de objetos no medibles a medibles.

El análisis de contenido, como aclara Guerrero (2002) “se basa en la lectura como instrumento de recogida de la información. Por lo tanto debe realizarse de modo científico: de manera sistemática, objetiva, replicable y válida”.

\section{El enfoque cualitativo.}

En el enfoque cualitativo de investigación a veces referido como investigación naturalista, fenomenológica, interpretativa o etnográfica, es una especie de "paraguas" en el cual se incluye una variedad de concepciones, visiones, técnicas y estudios no cuantitativos (Grinnell, 1997). Sus características más relevantes son:

1. EI investigador plantea un problema, pero no sigue un proceso claramente definido. Sus planteamientos no son tan específicos como en el enfoque cuantitativo.

Enfoque cualitativo Utiliza la recolecci6n de datos sin medición numérica para descubrir o afinar preguntas de investigación en el proceso de interpretación.

2. Se utiliza primero para descubrir y refinar preguntas de investigación (Grinnell, 1997).

3. Bajo la búsqueda cualitativa, en lugar de iniciar con una teoría particular y luego "voltear" al mundo empírico para confirmar si esta es apoyada por los hechos, el investigador comienza examinando el mundo social y en este proceso desarrolla una teoría coherente con 10 que observa que ocurre -con frecuencia denominada teoría fundamentada (Esterberg, 2002)-. Dicho de otra forma, las investigaciones cualitativas se fundamentan más en un proceso inductivo (explorar y describir, y luego generar perspectivas teóricas).

4 En la mayoría de los estudios cualitativos no se prueban hipótesis, estas se generan durante el proceso y van refinándose conforme se recaban más datos 0 son un resultado del estudio.

5. EI enfoque se basa en métodos de recolección de datos no estandarizados. No se efectiva una medición numérica, por 10 cual el an3.lisis no es estadístico. La recolección de los datos consiste en obtener las perspectivas y puntos de vista de los participantes (sus emociones, experiencias, significados y otros aspectos subjetivos).

Dentro de un análisis del contenido es muy importante destacar que los tres principios empleados por Bardin, le hacen ver la coherencia que debe de tener una información al ser obtenida por medio del buen uso de técnicas, asociando lo que describe clemente y santalla al momento de comprobar la hipótesis con el que quiere saber quién lo desea saber y para que lo desea saber, se comprende que en este momento después del empleo de las técnicas el investigador puede decir con exactitud que si la hipótesis que se había planteado dan los resultados esperados o lo in esperados (Bardin,1996; Clemente \& Santalla, 1991).

Rodríguez, J. M. (2011) nos argumenta que se pueda afirmar que la investigación forma parte del conocimiento humano en general y por tanto el conocimiento se ha definido como un proceso en el cual se relacionan un sujeto cognoscente (quien conoce) con un objeto de conocimiento (aquello que se conoce) lo que da como resultado un producto mental nuevo, llamado conocimiento. Así, el mismo término designa al proceso y al resultado de dicho proceso; es decir, llamamos conocimiento tanto a la operación subjetiva que lo produce, como al mismo producto. 


\section{Conclusiones}

El trabajo de investigación sobre los proceso metodológicos que conllevan las investigaciones se puede concluir que este estudio es de vital importancia, porque permitirá adquirir conocimientos referidos a las estrategias metodológicas que se aplican en los procesos investigativos y aplicarlos para la formación académica de los jóvenes que presentan dificultades educativas, asociadas a la deserción escolar de los colegios de secundaria del municipio de Somotillo departamento de Chinandega (Goode \& Hatt, 1991).

Los docentes en su mayoría tienen dominio e implementan estrategias de enseñanza para el aprendizaje, pero son estrategias propias de la educación regular secundaria y el currículo de la educación. Por lo tanto se hace necesario que los docentes simultáneamente sean capacitados en estrategias específicas para atender a la diversidad, como es el caso de uso adecuado de los diferentes materiales y métodos mejorando de esta manera el proceso de enseñanza aprendizaje.

\section{REFERENCIAS}

BOGDAN, R. y TAYOR, S.J. (1994): Introducción a los métodos cualitativos de investigación.

Bardin, L. (1996). Análisis de contenido (César Suárez, trad.). Madrid: Ed. Akal.

Clemente, M., \& Santalla, Z. (1991). El documento persuasivo: análisis de contenido y publicidad. Ed. Deusto.

González Río, M. J. (1997). Metodología de la investigación social. Alicante: Aguaclara.

Goode, J. y Hatt, P. (1991): Métodos de investigación social. México, Trillas.

Morgan, G. y Smircich, L. (1980): “The case for qualitative research”, en Academy of management review, n 5, pp. 491-500. .

Onwuegbuzie, A. J., Leech, N. L., Dickinson, W. B., \& Zoran, A. G. (2011). Un marco cualitativo para la recolección y análisis de datos en la investigación basada en grupos focales. Paradigmas: una revista disciplinar de investigación, $3(2), 127-157$.

Rodríguez, J. M. (2011). Métodos de investigación cualitativa. Revista de Investigación Silogismo, 1(08)

Sampieri, R., Collado, C. F., \& Lucio, P. B. (2010). Metodología de la Investigación. México: El Comercio SA.

Torres, M., Paz, K., \& Salazar, F. G. (2006). Métodos de recolección de datos para una investigación. Rev. Electrónica Ingeniería Boletín, 3, 12-20.

Wildemuth, B. M. (1993). Post-positivist research: two examples of methodological pluralism. The Library Quarterly, 63(4), 450-468.

Tesch, R (1990). Quantitative Research: Analysis types and software tools. Hampshire Falmer: press.

Grinnell, R. (1997). Social work research \& evaluation: Quantitative and qualitative approaches. Illinois: Peacock publishers.

Esterberg, K. G. (2002). Qualitative methods in social research. New York, NY, EE. UU.: McGraw-Hill.

Guerrero, C. S. (2002). Los entornos virtuales de aprendizaje como instrumento de mediación. Education in the Knowledge Society (EKS), 4(1). 